\title{
Aerodynamic Dispersion of Anode Arc Attachment Through a Converging-Diverging Nozzle
}

\author{
He-Ji Huang ${ }^{\circledR}$, Wen-Xia Pan, and Cheng-Kang Wu
}

\begin{abstract}
Electrode ablation is a key factor that affects the life and performance of the direct current arc plasma generator, which is closely related to the near-electrode behavior of the arc. Compared with the constricted-type arc root, diffusedtype arc root is more conducive to reduced thermal load, thereby improving the electrode life and the discharge stability. The attachment behavior of the arc root is related to the transport properties of the arc near the electrode wall and the aerodynamic and electromagnetic forces on the root of the arc. From this point of view, aerodynamical dispersion of the arc root using a converging-diverging nozzle was proposed, and the key factors that affect the dispersion were analyzed and verified.
\end{abstract}

Index Terms-Aerodynamical dispersion, arc plasma generator, diffused arc root attachment.

\section{NOMENCLATURE}

Primary Symbols

A Area.

$C \quad$ Specific heat.

$C_{d}$ Drag coefficient.

$E$ Energy.

$F \quad$ Force.

I Current.

$j \quad$ Current density.

$M \quad$ Mach number.

$m$ Mass flowrate.

$p$ Pressure.

$\rho$ Density.

$r \quad$ Radius.

$T$ Temperature.

$U$ Voltage.

$V \quad$ Volume.

$v \quad$ Velocity.

$\gamma \quad$ Ratio of specific heats.

$\eta \quad$ Efficiency.

$\theta$ Angle.

Manuscript received November 30, 2017; revised March 27, 2018; accepted July 10, 2018. This work was supported by the National Natural Science Foundations of China under Grant 11735004 and Grant 11575273. The review of this paper was arranged by Senior Editor J. Verboncoeur. (Corresponding author: He-Ji Huang.)

The authors are with the State Key Laboratory of High Temperature Gas Dynamics, Institute of Mechanics, Chinese Academy of Sciences, Beijing 100190, China, and also with the School of Engineering Science, University of Chinese Academy of Science, Beijing 100049, China (e-mail: huang@imech.ac.cn).

Color versions of one or more of the figures in this paper are available online at http://ieeexplore.iee.org.

Digital Object Identifier 10.1109/TPS.2018.2880246 $\mu_{0} \quad$ Vacuum permeability.

$\varphi \quad$ Polar angle.

\begin{tabular}{ll}
\multicolumn{2}{c}{ Notations } \\
0 & Initial/edge/wall value. \\
$*$ & Critical value. \\
$c$ & Arc column. \\
cond & Conduction. \\
$d$ & Dynamic. \\
drag & Drag. \\
$e$ & Expansion. \\
ero & Erosion. \\
in & Input. \\
max & Maximum value. \\
mean & Mean value. \\
$r$ & Radial direction. \\
rad & Radiation. \\
$s$ & Statistic. \\
$t$ & Throat/total. \\
$z$ & Axial direction.
\end{tabular}

\section{INTRODUCTION}

$\mathbf{T}$ HE increase of the number and scale of technical applications of arc plasma leads to more stringent requirements on the reliability of the process [1]. Among various influencing factors, thermal-induced electrode erosion is a key one. Electrode erosion not only leads to shorter lifetime of the plasma torch but also deteriorates the quality of the plasma process in a relatively uncontrollable way, which significantly reduces the cost-performance ratio. Looking back over the past decades of arc plasma torch developments, we can see that a continuous effort was devoted to improve the electrode erosion performance [2], [3].

From an energy balance consideration, the erosion rate of a certain volume of electrode material lying under the hot spot of an arc root can be written as

$$
V_{\text {ero }}=\frac{E_{\text {in }}-E_{\text {cond }}-E_{\text {rad }}}{\rho C \Delta T} .
$$

Equation (1) means that if the input energy $\left(E_{\text {in }}\right)$ could not be fully dissipated by the sum of thermal conduction ( $\left.E_{\text {cond }}\right)$ and radiation $\left(E_{\mathrm{rad}}\right)$, there must be some material removal $\left(V_{\text {ero }}\right)$ due to the temperature increase $\Delta T$ great enough to evaporate the material to maintain the energy balance. Among all the variables on the right hand of (1), $E_{\text {in }}$, which is proportional to $j^{2}$, might have the largest adjustable range. 


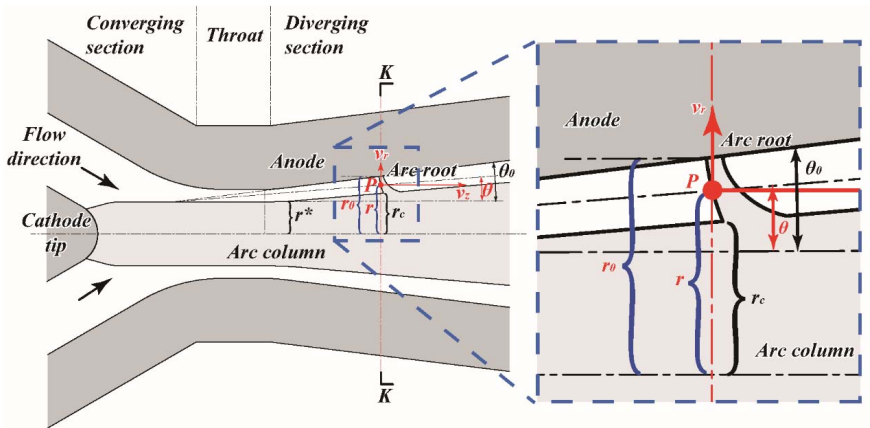

Fig. 1. Illustration of a nontransferred direct current plasma in a conical-shaped CD nozzle configuration. A partial enlarged view of the arc root (right).

As $j$ is defined by

$$
j=\frac{I}{A} .
$$

There are two ways to reduce $j$ by either reducing current $I$ or enlarging the arc attachment area $A$. The former could be realized by operating the arc plasma torch at a high-voltage and low-current mode, usually be possible with the addition of an interelectrode channel to elongate the arc length [4]-[6]. While for the latter one, either a rapid moving arc or a splitted electrical discharge could enlarge the temporal-averaged $A$ [7]-[11]. On the other hand, a stable but real diffusion attachment with transient larger $A$ is more desirable. This is because the moving arc might lead to high-frequency fluctuations which hinder its application in the advanced industry where high controllability is required.

However, although a diffusion attachment is observable in cases such as those when argon or natural gas (propane butane) exists [12], [13] or where a "hot" anode [14] is present, the mechanism of the diffusion attachment is still not clear.

Based on a qualitative understanding, the formation of a diffusion attachment is the result of the balance between the z-pinch of the arc root and the energetically favorable arc root shape and position following Steenbeck's principle of minimum power in gas discharge [15]. Both higher electrode temperature and plasma gas with higher electrical conductivity promote the electrical conductivity in the boundary layer, which reduces the energy barrier for arc breakdown, facilitating diffusion attachment. From this point of view, we propose a mechanism of arc root dispersion by improving the effect of aerodynamic expansion using a converging-diverging (CD) nozzle.

\section{TheOretiCAL ANAlysis AND RESUlts}

Fig. 1 illustrates the part of a nontransferred direct current plasma in a CD nozzle near the throat. The proposed $C D$ nozzle is in a conical shape, and the whole nozzle also acts as the anode. The cathode is inserted into the converging cavity. The expansion half-angle of the diverging part is $\theta_{0}$. A constricted anode attachment of the arc root is shown here for the theoretical analysis.

When the plasma flows through the throat and enters the diverging section, it will expand due to the pressure difference

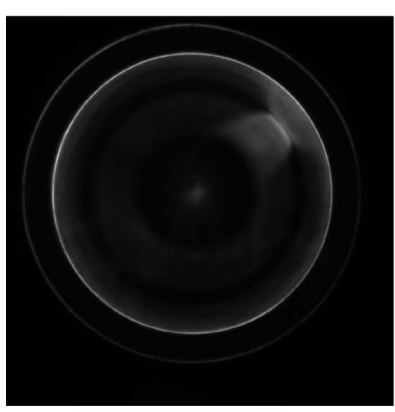

(a)

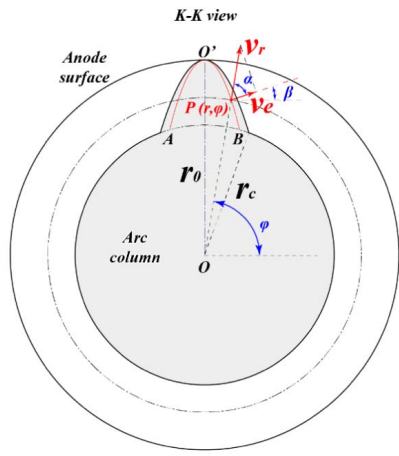

(b)
Fig. 2. (a) Anode arc attachment in a parabolic shape. (b) Schematics of the arc attachment on the $K-K$ plane.

between the nozzle inlet and outlet. The expansion angle $\theta$ of the flow varies at different radial positions. For point $P$ on the arc column, it seems to be reasonable to assume that

$$
\theta=\frac{r-r^{*}}{r_{0}-r^{*}} \theta_{0}
$$

where $r$ is the radial position of point $P, r^{*}$ is the radius of the arc column in the throat, and $r_{0}$ is the radius of the nozzle channel on the cross-sectional view of $K-K$, as illustrated in Figs. 1 and 2(b). Equation (3) assumes that the flow near the central axis does not expand much, while the expansion angle of the peripheral flow increases gradually until it equals $\theta_{0}$ at the nozzle inner wall. Such an expansion leads to a radial component of the flow velocity $\overrightarrow{\boldsymbol{v}}_{\mathbf{r}}=\overrightarrow{\boldsymbol{v}}_{z} \tan \theta$.

Fig. 2 shows the schematic arc root attachment on $K-K$ plane. Previous research shows that when the arc root attachment is a constricted one in a weakly expanded channel, it shows a parabolic shape of the arc column near the anode surface [8], as shown in Fig. 2(a). The plasma was generated at the arc current of $80 \mathrm{~A}$. The $10 \mathrm{vol} \% \mathrm{H}_{2}-\mathrm{Ar}$ with total gas flow rates of $8.6 \mathrm{slm}$ was used as a plasma gas. The chamber pressure was kept at $10 \mathrm{kPa}$. In Fig. 2(b), the radial velocity $\overrightarrow{\boldsymbol{v}}_{\mathbf{r}}$ will have another component of $\overrightarrow{\boldsymbol{v}}_{\mathbf{e}}$, which is normal to the parabola $A O^{\prime} B$ at point $P$. From the geometry relationship between vector components, the ratio of $\overrightarrow{\boldsymbol{v}}_{\mathbf{e}}$ to the main flow velocity $\overrightarrow{\boldsymbol{v}}$ at point $P$ is deduced as

$$
\frac{\overrightarrow{\boldsymbol{v}}_{\mathbf{e}}}{\overrightarrow{\boldsymbol{v}}_{z}}=\tan \left(\frac{r_{s}-a b}{1-a b} \theta_{0}\right) \cos \left[\varphi-\arctan \left(-\frac{1}{2} \frac{b}{k r_{t} r_{s} \cos \varphi}\right)\right]
$$

where $r_{s}=\left(r / r_{0}\right)$ is the radius position normalized by the nozzle radius. The coefficients are $a=\left(r^{*} / r_{t}\right), b=\left(r_{t} / r_{0}\right)$, and $k=-\left(r_{0}-r \sin \varphi / r^{2} \cos ^{2} \varphi\right)$. Note that $\overrightarrow{\boldsymbol{v}}_{z}$ is a function of position $r, \overrightarrow{\boldsymbol{v}}_{z}=\overrightarrow{\boldsymbol{v}}_{z}(r)$. For a given point $P$ with coordinates of $\left(r_{p}, \varphi_{p}\right)$, the parabola of $A O^{\prime} P B$ is determined by

$$
r \sin \varphi=k_{P} r^{2} \cos ^{2} \varphi+r_{0} .
$$

From (4), the ratio of $v_{e} / v_{z}$ is calculated for discrete points in the parabola region, as shown in Fig. 3(a) and (b). Fig. 3 shows the distributions of $v_{e} / v_{z}$ on the up-right quarter of the $K-K$ view, as illustrated in Fig. 2(b). In this example, $\theta_{0}$ equals $7^{\circ}$ and the relative arc column size is $70 \%$ of the whole channel $(a=0.7)$. Following the assumption in (3), 

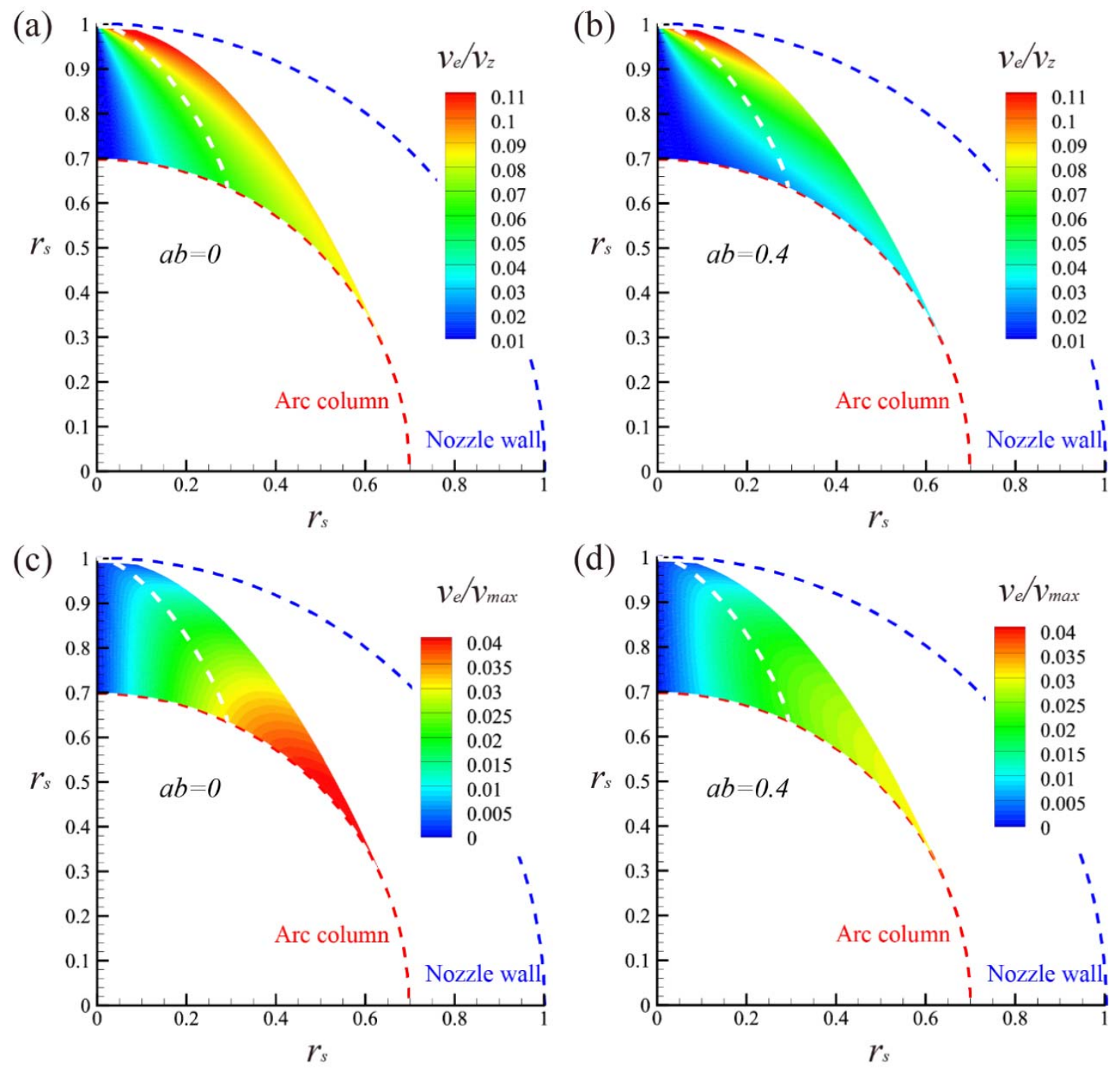

Fig. 3. Component $v_{e}$ distributions in the parabolic region where $\theta_{0}=7^{\circ}$. (a) and (c) $a b=0$. (b) and (d) $a b=0.4$. (c) and (d) $v$ follows a parabolic distribution.

for a larger $a b\left(a b=r^{*} / r_{0}\right)$, the value of component $v_{r}$ is smaller, which is more pronounced near the arc column center. Fig. 3(a) and (b) shows the comparison of $a b=0$ and 0.4 , respectively. When the considered point is closer to the parabolic axis of symmetry, the direction of $\overrightarrow{\boldsymbol{v}}_{\mathrm{r}}$ is also closer to the tangential direction, resulting in a smaller normal component of $\overrightarrow{\boldsymbol{v}}_{\mathrm{e}}$, which is clearly shown in Fig. 3.

If the main velocity also follows a parabolic distribution radially, which is usually consistent with the experimental results, that is, $v_{z}=v_{\max }\left(1-r_{s}^{2}\right)$. When the flow is axisymmetric, $v_{\max }=2 v_{\text {mean }}$, thus (4) can be rewritten to

$$
\begin{aligned}
& \frac{v_{e}}{v_{\text {mean }}}=2\left(1-r_{s}^{2}\right) \tan \left(\frac{r_{s}-a b}{1-a b} \theta_{0}\right) \\
& \times \cos \left[\varphi-\arctan \left(-\frac{1}{2} \frac{b}{k r_{t} r_{s} \cos \varphi}\right)\right] .
\end{aligned}
$$

Fig. 3(c) and (d) shows the distribution of $v_{e} / v_{\max }$, which is the result of a counterbalance between the value of $v$ and $v_{r} / v$.

In order to realize the dispersion of the arc root, the aerodynamic drag force should be large enough to overcome the pinch effect caused by the electromagnetic force and to expand and push the arc column region close enough to the anode surface to achieve breakdown. The aerodynamic drag force

$$
F_{\text {drag }}=p_{d} C_{d} A_{a} .
$$

The dynamic pressure $p_{d}$

$$
p_{d}=\frac{1}{2} \rho v^{2}=\frac{1}{2} p_{s} \gamma M^{2} .
$$

Based on the relationships between $p_{s}$ and $p_{t}, \rho_{s}$ and $\rho_{t}$, and $T_{s}$ and $T_{t}$ for compressible flows [16], together with the mass conservation equation, $p_{d}$ can be rewritten to

$$
p_{d}=\frac{1}{2} \frac{\dot{m}}{A} \sqrt{\gamma R T_{t}} M\left(1+\frac{\gamma-1}{2} M^{2}\right)^{\frac{-1}{2}} .
$$

Let $K_{d}=M\left(1+(\gamma-1 / 2) M^{2}\right)^{(-1 / 2)}$. Fig. 4 shows the dependence of $K_{d}$ on $M$ for various $\gamma$. It shows that $K_{d}$ increases with $M$, and the effect of $\gamma$ is quite small at low $M$ conditions. When $M$ is less than $0.5, K_{d}$ increases almost linearly with $M$. This is because although (8) suggests that $p_{d}$ increases with $M^{2}$, the statistic pressure $p_{s}$ drops rapidly at higher flow velocity. Therefore, the portion of the 


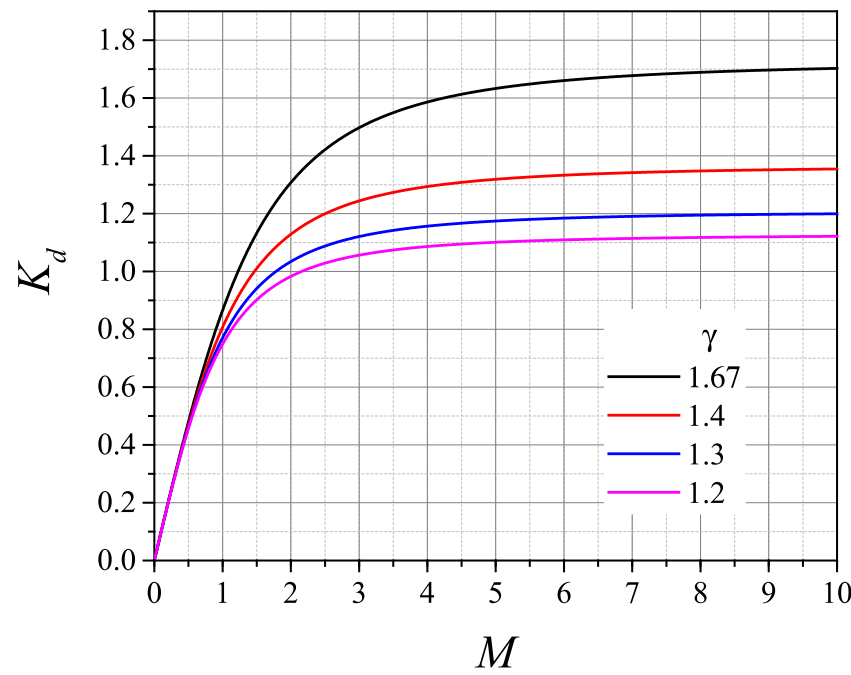

Fig. 4. Dependence of $K_{d}$ on $M$ for various $\gamma$.

expansion component of the drag force is similar to that shown in (4) and (6)

$$
\begin{aligned}
& \frac{p_{\mathrm{de}}}{p_{d}}=2\left(1-r_{s}^{2}\right) \tan \left(\frac{r_{s}-a b}{1-a b} \theta_{0}\right) \\
& \times \cos \left[\varphi-\arctan \left(-\frac{1}{2} \frac{b}{k r_{t} r_{s} \cos \varphi}\right)\right] .
\end{aligned}
$$

From the expression of $K_{d}$, it is shown that when $M$ is much greater than $1, K_{d}$ approaches the value of $(\gamma-1 / 2)^{(-1 / 2)}$. This is because with certain enthalpy level, the dynamic pressure has its maximum when all the energies have been converted to kinetic energy, which will never exceed the total energy of the flow.

The total temperature is contributed by two parts: the cold flow temperature and the temperature rise due to the electric power input. It can be written as

$$
T_{t}=\eta \frac{I U}{C_{p} \dot{m}}+T_{0}
$$

When the plasma exists, since $T_{0} / T_{t} \ll 1, T_{0}$ could be neglected, and therefore (8) is approximated to

$$
p_{d}=\frac{1}{2 A} \sqrt{I U \dot{m} \eta(\gamma-1)} K_{d} .
$$

Although (12) is only valid for calorically perfect gas, when the change of $\gamma$ is taken into account, it can be used for approximate estimations with acceptable error range at low Mach number cases (e.g., $M<2$ ).

To simplify the estimation of the $z$-pinch effect of the arc attachment, all the cross sections perpendicular to $\vec{j}$ are assumed to be in circular shapes, as shown in Fig. 5. In this case, if current $I$ is in the parabolic distribution, the $z$-pinch pressure of a point of the arc column in a circular cross section of radius $r_{z}$ equals

$$
p_{z}=\frac{5}{3} \frac{\mu_{0}}{4 \pi} \frac{I^{2}}{2 \pi r_{z}^{2}\left(1-\sin \varphi_{0}\right)}\left(1-\frac{12}{5} \frac{r_{1}^{2}}{r_{z}^{2}}+\frac{9}{5} \frac{r_{1}^{4}}{r_{z}^{4}}-\frac{2}{5} \frac{r_{1}^{6}}{r_{z}^{6}}\right) .
$$
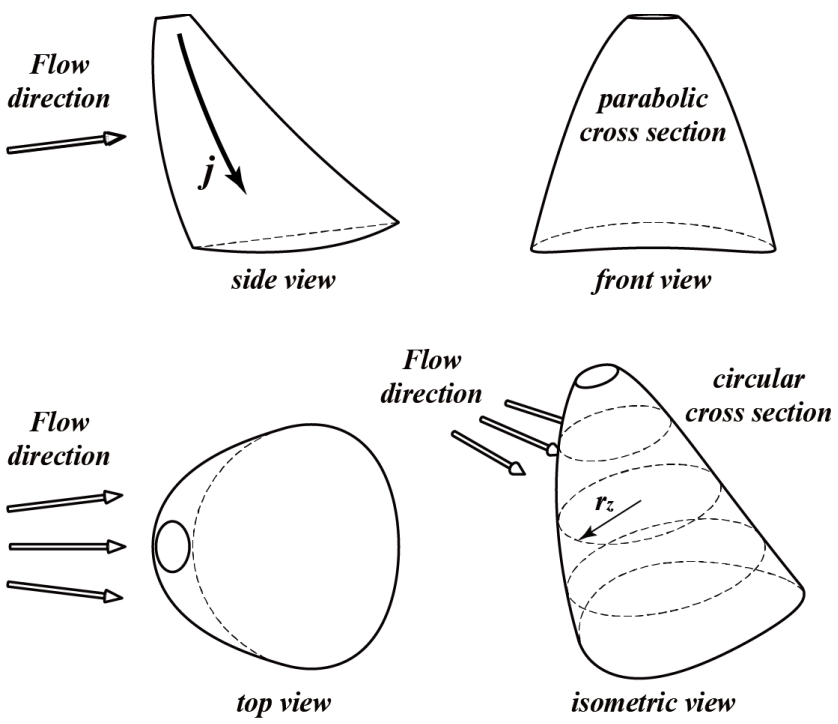

Fig. 5. Proposed 3-D arc column geometry near the anode surface.

Here, $r_{1}=r \cos \varphi$, and $\varphi_{0}$ represents the position of the arc column edge.

From the combination of (10), (12), and (13), it shows that the proportional relationship between the $z$-pinch pressure and the expansion component of dynamic pressure is

$$
\frac{p_{z}}{p_{\mathrm{de}}} \propto \frac{\mu_{0} I^{2}}{\sqrt{I U \dot{m} \eta}} \frac{1}{K_{d}}
$$

which suggests that the arc column tends to expand at higher gas flow rates, higher Mach number, and higher power input efficiency, while with higher arc current, the $z$-pinch effect is more pronounced.

Fig. 6(a)-(d) shows the effect of $M$ on $\left(p_{z} / p_{\text {de }}\right)$ with other parameters the same as those illustrated in Fig. 3. The dark region in Fig. 6 shows $p_{z}$ being greater than $p_{\text {de }}$. However, this does not mean that the arc column inside will not expand, because the $z$-pinch pressure itself is somewhat already balanced by the pressure caused by the temperature difference between the inner and outer arc columns. The extra $p_{\mathrm{de}}$, even if it is smaller than $p_{z}$, also causes the arc column near the anode surface to expand. The light gray parts shown in Fig. 6 are $p_{z}$ being smaller than $p_{\text {de }}$. Within this region, the arc will swell rapidly, especially at the outer edge. Once the edge of the arc column expands to such a position that the breakdown voltage $V_{b}$ is smaller than the original voltage drop $V_{o}$ (as illustrated in the inset of Fig. 6), a wider arc attachment will form and eventually get a diffusion attachment. In Fig. 6, it is clearly shown that $M$ plays an important role for the dispersion of the arc root. When $M$ increases from 0.01 to 0.6 , the area of $p_{\text {de }}$ larger than $p_{z}$ increases from $6.8 \%$ to $51.7 \%$.

Some preliminary experimental results have been shown in [17]. Using a torch with $r_{t}=3 \mathrm{~mm}$ and $\theta_{0}=5^{\circ}, \mathrm{N}_{2}$ plasma was generated in vacuum at flow rates among 4.5-30 slm. A self-designed copper mirror with weak-reflecting material imbedded at the center part was used to observe the condition of the arc root attachment on the anode surface. The images of the arc root attachments were recorded by an intensified charge coupled device camera at an exposure time of $5 \mu \mathrm{s}$. The 

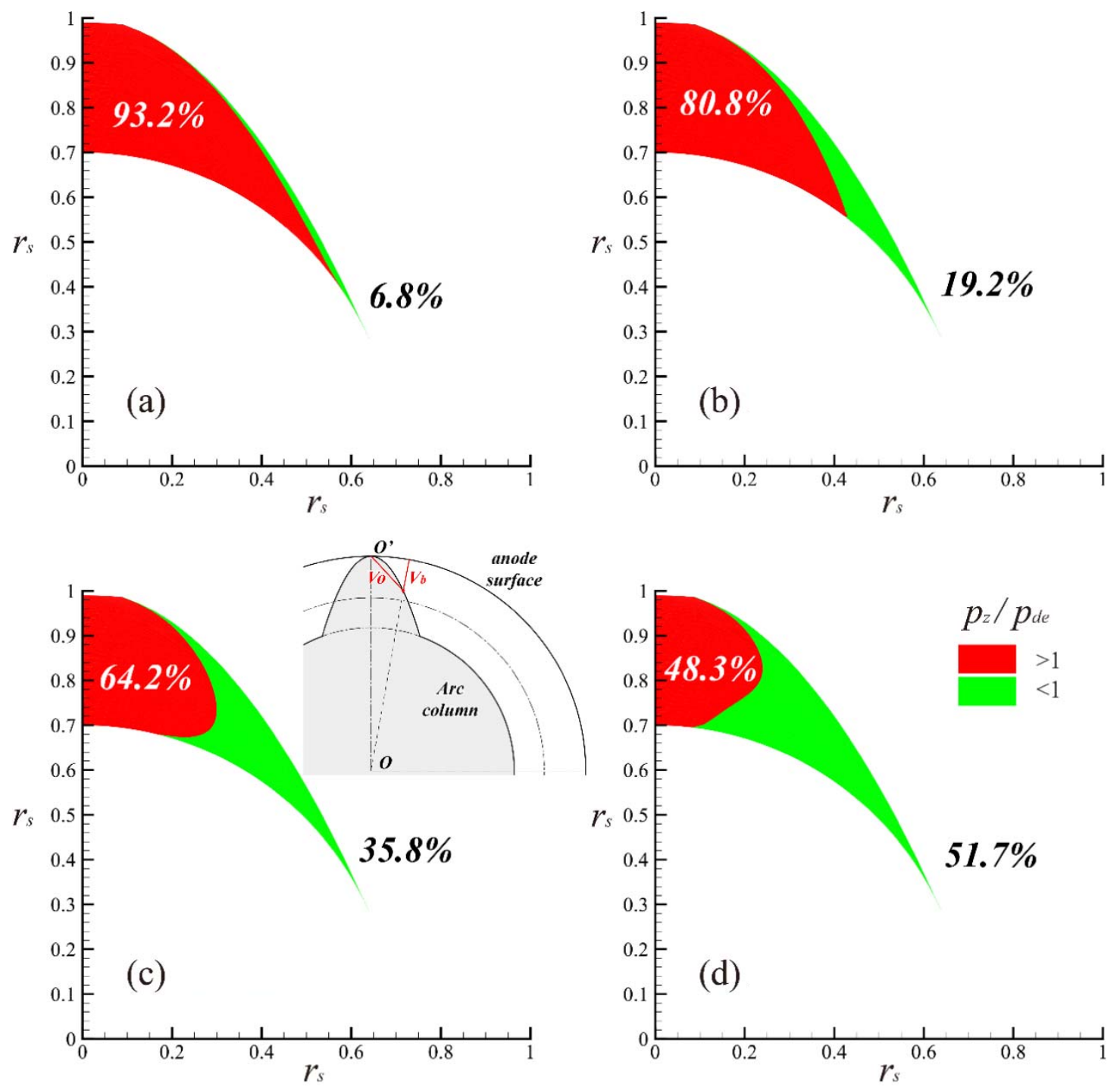

Fig. 6. Effect of $M$ on the ratio of $p_{z} / p_{\text {de }}$. (a) $M=0.01$. (b) $M=0.1$. (c) $M=0.3$. (d) $M=0.6$.

TABLE I

Experimental Parameters and the Observed Transition of ARC AtTachment Types

\begin{tabular}{|l|l|l|l|l|l|}
\hline$I(\mathrm{~A})$ & $U(\mathrm{~V})$ & $\dot{m}(\mathrm{~kg} / \mathrm{s})$ & $\frac{\mu_{0} I^{2}}{\sqrt{I U \dot{m}}}$ & $p_{c}(\mathrm{~Pa})$ & Arc attachment type \\
\hline 120 & 25 & $0.12 \mathrm{E}-03$ & $2.96 \mathrm{E}-02$ & 50 & constriction \\
\hline 140 & 45 & $0.14 \mathrm{E}-03$ & $2.58 \mathrm{E}-02$ & 74 & transition \\
\hline 140 & 55 & $0.18 \mathrm{E}-03$ & $2.08 \mathrm{E}-02$ & 85 & diffusion \\
\hline
\end{tabular}

transition from constriction to diffusion arc attachment is seen when the total plasma gas flow rate increases. Table I shows the discharge parameters and the corresponding observed arc root attachment types. Although $M$ was not measured in the experiment, a higher $M$ and thus $K_{d}$ at elevated gas flow rate is expected, because the pressure in the cathode cavity $p_{c}$ increases. It is shown that when the dimensionless value of $\left(\mu_{0} I^{2} /(I U \dot{m})^{1 / 2}\right)$ decreases from $2.96 \mathrm{E}-2$ to $2.08 \mathrm{E}-2$, the arc attachment type changes from constriction to diffusion one.

\section{CONCLUSION}

Above results suggest that it is possible to achieve a diffusion arc attachment by optimizing the geometry parameters of the CD nozzle, such as the throat size and the expansion angle. To get a desired $M$ large enough for the dispersion of 
the anode arc root attachment, or with a certain CD nozzle configuration, a combination of the discharge parameters can also affect the arc attachment type. A dimensionless parameter of $\left(\mu_{0} I^{2} /(I U \dot{m})^{1 / 2}\right)$ could be utilized to estimate the arc attachment type in present experimental conditions.

\section{REFERENCES}

[1] M. F. Zhukov and I. M. Zasypkin, Thermal Plasma Torches: Design, Characteristics, Applications. Cambridge, U.K.: Cambridge International Science Publishing, 2007.

[2] Y. Wu, "Visualization and mechanisms of splashing erosion of electrodes in a DC air arc," J. Phys. D: Appl. Phys., vol. 50, no. 47, p. 47LT01, Nov. 2017.

[3] M. Tanaka, T. Hashizume, K. Saga, T. Matsuura, and T. Watanabe, "Diode-rectified multiphase AC arc for the improvement of electrode erosion characteristics," J. Phys. D: Appl. Phys., vol. 50, no. 46, p. 465604 , Oct. 2017.

[4] M. Gindrat, R. K. Schmid, R. Molz, and D. Hawley, "Recent and advanced techniques to increase reliability and life of torches in the thermal spray industry," in Proc. 3rd Int. Round Table Thermal Plasmas Ind. Appl., Muldersdrift, South Africa, 2011, p. 71.

[5] W. Pan, X. Meng, C. Wu, and X. Chen, "Experimental study on the thermal argon plasma generation and jet length change characteristics at atmospheric pressure," Plasma Chem. Plasma Process., vol. 26, no. 4, pp. 335-345, Aug. 2006.

[6] W. Pan, Z. Y. Guo, X. Meng, H. J. Huang, and C. K. Wu, "Fluctuation characteristics of arc voltage and jet flow in a non-transferred dc plasma generated at reduced pressure," Plasma Sour. Sci. Technol., vol. 18, no. 4, p. 045032, Oct. 2009

[7] J. P. Trelles, "Computational study of flow dynamics from a dc arc plasma jet," J. Phys. D: Appl. Phys., vol. 46, no. 25, p. 255201, Jun. 2013.
[8] H.-J. Huang, W. X. Pan, and C. K. Wu, "Arc root motions in an argon-hydrogen direct-current plasma torch at reduced pressure," Chin. Phys. Lett., vol. 25, no. 11, p. 4058, Nov. 2008.

[9] L. C. Li, W. D. Xia, H. L. Zhou, Z. P. Zhou, and B. Bai, "Experimental observation and numerical analysis of arc plasmas diffused by magnetism," Eur. Phys. J. D, vol. 47, no. 1, pp. 75-81, Apr. 2008.

[10] O. M. Chumak, A. Mašláni, and Hrabovský, "Experimental observations of arc-anode attachment in steam-argon-air environment," in Proc. 12th High-Tech Plasma Process. Conf., vol. 406, 2012, p. 012009.

[11] J. Schein, J. Zierhut, M. Dzulko, G. Forster, and K. D. Landes, "Improved plasma spray torch stability through multi-electrode design," Contrib. Plasma Phys., vol. 47, no. 7, pp. 498-504, Nov. 2007.

[12] M. Auweter-Kurtz, H. L. Kurtz, and S. Laure, "Plasma generators for re-entry simulation," J. Propuls. Power, vol. 12, no. 6, pp. 1053-1061, 1996.

[13] P. Wenxia, M. Xian, L. Teng, C. Xi, and W. Chengkang, "Comparative observation of $\mathrm{Ar}, \mathrm{Ar}-\mathrm{H}_{2}$ and $\mathrm{Ar}-\mathrm{N}_{2} \mathrm{DC}$ arc plasma jets and their arc root behaviour at reduced pressure," Plasma Sci. Technol., vol. 9, no. 2 p. 152, Apr. 2007.

[14] W. Pan, H. Huang, and C. Wu, "Effect of nozzle temperature on the performance of a $1 \mathrm{~kW} \mathrm{H}-\mathrm{N}_{2}$ arcjet thruster," Plasma Sci. Technol., vol. 12, no. 4, pp. 473-477, 2010.

[15] H.-P. Li, E. Pfender, and X. Chen, "Application of Steenbeck's minimum principle for three-dimensional modelling of DC arc plasma torches," J. Phys. D: Appl. Phys., vol. 36, no. 9, pp. 1084-1096, Apr. 2003.

[16] AMES Research Staff, "Equations, tables, and charts for compressible flow," Ames Aeronautical Lab., Moffett Field, CA, USA, Tech. Rep. $1135,1953$.

[17] W. Pan, L. Chen, X. Meng, Y. Zhang, and C. Wu, "Sufficiently diffused attachment of nitrogen arc by gasdynamic action," Theor. Appl. Mech. Lett., vol. 6, no. 6, pp. 293-296, Nov. 2016.

Authors' photographs and biographies not available at the time of publication. 